\title{
What triggers galaxy transformations? The environments of post-starburst galaxies
}

\author{
David W. Hogg ${ }^{1,2}$, Morad Masjedi ${ }^{1}$, Andreas A. Berlind ${ }^{1}$, Michael R. Blanton ${ }^{1}$, \\ Alejandro D. Quintero ${ }^{1}$, J. Brinkmann ${ }^{3}$
}

\begin{abstract}
Star-formation history is strongly related to environment; the most massive and least star-forming galaxies reside in the highest density environments. There are now good observational reasons to believe that the progenitors of these red galaxies have undergone starbursts, followed by a post-starburst phase. Poststarburst ("K+A" or "E+A") galaxies appear in the SDSS visible spectroscopic data by showing an excess of A star light (relative to $\mathrm{K}$ giant light) but deficient $\mathrm{H} \alpha$ line emission. We investigate the environments of these galaxies by measuring (1) number densities in $8 h^{-1}$ Mpc radius comoving spheres, (2) transverse distances to nearest Virgo-like galaxy clusters, and (3) transverse distances to nearest luminous-galaxy neighbors. We compare the post-starburst galaxies to currently star-forming galaxies identified solely by A-star excess or $\mathrm{H} \alpha$ emission. We find that post-starburst galaxies are in the same kinds of environments as starforming galaxies; this is our "null hypothesis". More importantly, we find that at each value of the A-star excess, the star-forming and post-starburst galaxies lie in very similar distributions of environment. Other studies finding similar results have argued that galaxy transformations occur slowly (time scales $>1$ Gyr), but this is at odds with the observational evidence that red galaxies are formed via starbursts. The only deviations from our null hypothesis are barely significant: a slight deficit of post-starburst galaxies (relative to the star-forming population) in very low-density regions, a small excess inside the virial radii of clusters, and a slight excess with nearby neighbors. None of these effects is strong enough to make the post-starburst galaxies a high-density phenomenon, or to argue that the starburst events are primarily triggered by external tidal impulses (e.g., from close passages of massive galaxies). The small excess inside cluster virial radii
\end{abstract}

\footnotetext{
${ }^{1}$ Center for Cosmology and Particle Physics, Department of Physics, New York University, 4 Washington Place, New York, NY 10003

2 To whom correspondence should be addressed: david.hogg@nyu.edu

3 Apache Point Observatory, 2001 Apache Point Road, P.O. Box 59, Sunspot, NM 88349
} 
suggests that some post-starbursts are triggered by interactions with the intracluster medium, but this represents a very small fraction of all post-starburst galaxies.

Subject headings: galaxies: clusters: general — galaxies: evolution — galaxies: statistics — galaxies: stellar content — stars: formation

\section{Introduction}

How do old, dead, early-type galaxies form? There are two strong arguments that bulge-dominated galaxies (e.g., ellipticals, lenticulars, and very early-type spirals) have progenitors that went through a starburst phase. The first is that bulge-dominated galaxies show enhancements in $\alpha$-type elements over the Solar chemical abundance mix (e.g., Worthey 1998; Eisenstein et al. 2003). These abundance patterns are naturally produced when star formation - or the last phase of star formation - has occured in a burst too rapid to allow recycling of the elements ejected by Type 1a supernovae. The second argument is that the post-starburst galaxies identified spectrally in large surveys of the Local Universe have the colors, surface brightnesses, and radial profiles that would be expected if they are to evolve passively into new bulge-dominated galaxies (Quintero et al. 2004); they cannot evolve passively into disk-dominated or other late types. Starburst origin is also supported indirectly by the uniformity seen in early-type galaxies' stellar populations (e.g., Eisenstein et al. 2003) and their lack of large resevoirs of cold gas and dust.

Post-starburst galaxies are identified spectroscopically by having a large contribution to their spectral energy distribution from A stars - i.e., new stars must have formed within the last $\sim 1$ Gyr - but no, or very little, contribution from $\mathrm{O}$ and $\mathrm{B}$ stars - i.e., no new stars have formed within the last $\sim 0.01$ Gyr. In practice, these " $\mathrm{K}+\mathrm{A}$ " or "E+A" galaxies" (Dressler \& Gunn 1983; Zabludoff et al. 1996; Dressler et al. 1999) are identified by having strong Balmer absorption or blue continua (A-star indicators) but no $\mathrm{H} \alpha$ or [O II] emission lines (O and B-star indicators). Importantly, because A stars have known lifetimes, the evolution of the population can be "timed" and rates computed; we find that of order 1 percent of the galaxy population is going through this phase each Gyr at $z \sim 0.1$ (Quintero et al. 2004).

What is not known is what precedes or triggers the (necessarily rapid) truncation of

\footnotetext{
${ }^{1}$ The terminology " $\mathrm{K}+\mathrm{A}$ " is to be preferred to " $\mathrm{E}+\mathrm{A}$ " because the identification is spectral, not morphological, and "K" and "A" name spectral types. "E" names a morphological type (Franx 1993; Dressler et al. 1999).
} 
star-formation. Is it an external event, such as a tidal impulse, accretion event, or major merger? Or is it a purely internal event, such as an AGN flare or the abrupt exhaustion of star-formation fuel? Either way, since disk-dominated galaxies are the galaxies that contain young stars and the cold-gas fuel to make more, post-starburst galaxies must lie on some kind of evolutionary pathway between the disk-dominated and bulge-dominated populations.

The distribution of galaxy star-formation rates is a strong function of environment, with much lower star-formation rates in higher density regions (e.g., Kennicutt 1983; Hashimoto et al. 1998; Balogh et al. 2001; Martínez et al. 2002; Lewis et al. 2002; Gomez et al. 2003; Blanton et al. 2003b; Hogg et al. 2003, 2004; Kauffmann et al. 2004; Blanton et al. 2005; Quintero et al. 2006). Although it is customary to think of this as being a consequence of the morphology-density relation (Dressler 1980; Postman \& Geller 1984), in fact recent studies with large samples have shown that in fact the star-formation-environment relation has more explanatory or informative power than the morphology-density relation, at least with the morphological proxies currently available for large samples (Hashimoto et al. 1998; Hashimoto \& Oemler 1999; Kauffmann et al. 2004; Blanton et al. 2005; Quintero et al. 2006). How is the information about environment transmitted to galaxy star-formation activity? Are there violent events when galaxies fall into high density regions? Or do the galaxies reduce their star-formation rates gradually as they find themselves in denser and denser environments? Studies of the (strong, observed) evolution of the fraction of galaxies in clusters that are blue (often called the "Butcher-Oemler effect"; Butcher \& Oemler 1978) have generally concluded that this evolution is gradual, at least when compared to the lifetimes of A stars (Poggianti et al. 1999; Balogh et al. 2000; Kodama \& Bower 2001), although there are certainly some galaxies in clusters that appear to be undergoing rapid evolution (e.g., Vogt et al. 2004; Yang et al. 2004). It is not clear how to reconcile this conclusion of gradual evolution with the conclusion (mentioned above) that a typical earlytype galaxy has undergone a massive starburst in its past.

Galaxy star-formation rates are also evolving very rapidly with redshift in the field; this result comes from many different techniques at many different wavelengths (e.g., Lilly et al. 1996; Hammer et al. 1997; Rowan-Robinson et al. 1997; Hogg et al. 1998; Tresse \& Maddox 1998; Cowie et al. 1999; Flores et al. 1999; Mobasher et al. 1999; Haarsma et al. 2000; Juneau et al. 2005; Schiminovich et al. 2005). This is usually imagined as being related not to infall into dense regions but rather to the supply of cold gas. On the other hand, since gravitational clustering brings galaxies into more and more dense environments with cosmic time, this might not be unrelated to the star-formation-environment relation and the Butcher-Oemler effect.

At the same time as star formation in the Universe is declining, the total density of 
stellar mass on the "red sequence" of early-type or bulge-dominated galaxies is increasing (Bell et al. 2004; Blanton 2006; Faber et al. 2006; Brown et al. 2006). If blue galaxies transform into red galaxies via a post-starburst phase, as we believe they must, then this evolution of the red sequence must be quantitatively matched with an evolving population of post-starburst galaxies.

As transition objects between the star-forming, disk-dominated and dead, bulge-dominated populations, the post-starburst galaxies could in principle have the environmental characteristics of either. Originally, $\mathrm{K}+\mathrm{A}$ galaxies were found in high-density regions (Dressler \& Gunn 1983; Couch \& Sharples 1987), and thought to be a "cluster" population. Of course the early searches for such galaxies were made in cluster fields. Once systematic searches for $\mathrm{K}+\mathrm{A}$ galaxies were made in large redshift surveys, it was found that they are not particularly concentrated in clusters or high density regions, but rather live in a wide range of environments (Zabludoff et al. 1996; Quintero et al. 2004; Blake et al. 2004; Goto 2005, Helmboldt et al., in preparation). In the large SDSS and 2dFGRS samples, it can be shown that the mean environment (Quintero et al. 2004, Helmboldt et al., in preparation) and distribution of environments (Blake et al. 2004) are both similar to those of spiral or disk-dominated galaxies.

The environments of disk-dominated galaxies - isolation and small groups, where the virialized mass has a similar velocity dispersion to the contained galaxies - are the best environments in the Local Universe to find galaxy-galaxy mergers, which are the top candidates for triggers for the post-starburst galaxies. After all, the major mergers observed in the Local Universe are all associated with very high star-formation rates, and major mergers are expected to disrupt disks and leave behind the dynamically hot stellar orbits characteristic of the bulge-dominated population.

With a sample of more than $10^{3} \mathrm{~K}+\mathrm{A}$ galaxies (Quintero et al. 2004), we are in a position to ask much more detailed questions about the range of environments in which they lie, and the relationships between environmental and star-formation properties. That is the purpose of this Article, with the goal of constraining the possible triggering mechanisms for this very important galaxy population.

In what follows, a cosmological world model with $\left(\Omega_{\mathrm{M}}, \Omega_{\Lambda}\right)=(0.3,0.7)$ is adopted,

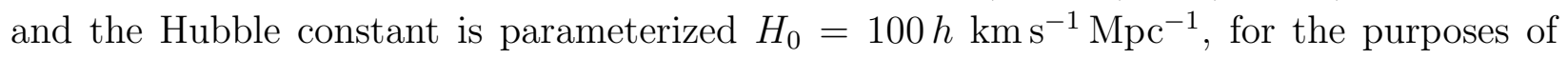
calculating distances(e.g., Hogg 1999). 


\section{Data}

The SDSS is taking ugriz CCD imaging of $10^{4} \mathrm{deg}^{2}$ of the Northern Galactic sky, and, from that imaging, selecting $10^{6}$ targets for spectroscopy, most of them galaxies with $r<17.77$ mag (Gunn et al. 1998; York et al. 2000; Stoughton et al. 2002; Abazajian et al. 2003; Abazajian et al. 2004).

All the data processing, including astrometry (Pier et al. 2003), source identification, deblending and photometry (Lupton et al. 2001), calibration (Fukugita et al. 1996; Smith et al. 2002; Ivezić et al. 2004), spectroscopic target selection (Eisenstein et al. 2001; Strauss et al. 2002; Richards et al. 2002), spectroscopic fiber placement (Blanton et al. 2003a), spectral data reduction and analysis (Schlegel \& Burles, in preparation, Schlegel in preparation) are performed with automated SDSS software.

Galaxy absolute magnitudes and colors are computed in fixed bandpasses, using Galactic extinction corrections (Schlegel et al. 1998) and $K$ corrections (computed with kcorrect v1_11; Blanton et al. 2003). They are $K$ corrected not to the redshift $z=0$ observed bandpasses but to bluer bandpasses ${ }^{0.1} g,{ }^{0.1} r$ and ${ }^{0.1} i$ "made" by shifting the SDSS $g, r$, and $i$ bandpasses to shorter wavelengths by a factor of 1.1 ( $c f$. ., Blanton et al. 2003; Blanton et al. $2003 b$ ). This means that galaxies at redshift $z=0.1$ (typical of the sample used here) have trivial (but non-zero) $K$ corrections.

We capitalize on the extremely good spectrophotometric calibration of the SDSS data and measure, for each galaxy, the excess light in each fiber spectrum in the wavelength range $3800<\lambda<5400 \AA$ coming from A-type stars relative to K-type stars, normalized to the mean spectrum of an old galaxy in the SDSS. This measurement is described elsewhere (Quintero et al. 2004); briefly, we perform a linear fit of the spectral section to a linear combination of the mean SDSS old galaxy spectrum (the "K" spectrum) and the mean SDSS A-star spectrum (the "A" spectrum) with the locations of possible emission lines masked out. The A-star excess is then the ratio $A / K$ of the amplitudes of the two spectral components from the fit. We also measure the line flux and equivalent width (EW) of the $\mathrm{H} \alpha$ line using the " $\mathrm{K}+\mathrm{A}$ " fit as a continuum model (which effectively removes the absorption contribution to the flux at $\mathrm{H} \alpha$ ). These measurements are performed exactly as described by us previously (Quintero et al. 2004).

The "units" in which the A-star excess is measured are arbitrary, but used here (where the units correspond to a luminosity ratio in the above-mentioned wavelength band) they can be calibrated by their relationship to $\mathrm{H} \alpha \mathrm{EW}$; typically

$$
\frac{A}{K} \sim \frac{\mathrm{H} \alpha \mathrm{EW}}{40 \AA}
$$


(Quintero et al. 2004). For the purposes of what follows, we define "low H $\alpha$ " galaxies to be those with $\mathrm{H} \alpha$ EW less than 1/8 the value implied by equation (1). We define star-forming "H $\alpha$ excess" galaxies to be those with $\mathrm{H} \alpha \mathrm{EW}>5.0 \AA$ and star-forming "A-star excess" galaxies to be those with $(A / K)>0.2$ in our arbitrary units. $\mathrm{K}+\mathrm{A}$ galaxies, the subject of this study, are those that are "low $\mathrm{H} \alpha$ " but "A-star excess"; i.e., they are star-forming according to the A-star excess, but not according to the $\mathrm{H} \alpha$ emission. These definitions are illustrated graphically in Figure 1.

Often, $\mathrm{K}+\mathrm{A}$ galaxies are found in surveys by their large $\mathrm{H} \delta$ absorption $\mathrm{EW}$, because that indicates A stars but little line emission to dilute or fill the absorption trough (Zabludoff et al. 1996; Blake et al. 2004; Goto 2005). These studies usually use other emission lines (such as $[\mathrm{O} I I]$ ) to veto star-forming galaxies, but they are less general than what is presented here, because they select only for post-starburst galaxies dominated by the A stars with the strongest $\mathrm{H} \delta$ lines, and they do not separate post-starburst galaxies out of the main galaxy population as cleanly as the method used here. On the other hand, the $\mathrm{H} \delta$ method can be used with poorly calibrated spectra, because it relies only on a local measure of the spectral shape around the $\mathrm{H} \delta$ line. Fortunately, with the SDSS spectra, spectrophotometric calibration is excellent (Quintero et al. 2004).

In principle it is possible for an actively star-forming object to appear post-starburst if the very young stars are fully dust-enshrouded but migrate out of the dusty regions on timescales of < 1 Gyr (Poggianti \& Wu 2000; Quintero et al. 2004; Blake et al. 2004). Though this is possible, even the dustiest starbursts known do emit significant $\mathrm{H} \alpha$, and indeed $20 \mathrm{~cm}$ radio observations of a sample of 36 SDSS post-starburst candidates find no evidence for significant, hidden star-formation (Goto 2004).

Around every galaxy a density $\rho_{8}$ is measured as described elsewhere (Blanton et al. 2003b; Hogg et al. 2003); briefly, it is a count of the number of neighbors in the SDSS spectroscopic sample inside a $8 h^{-1} \mathrm{Mpc}$ radius comoving sphere in comoving distance space (with no correction for redshift distortions), divided by the uniform-density predicted number, made from the galaxy luminosity function (Blanton et al. 2003c) and the SDSS window function, to make a dimensionless density. The sample used to infer $\rho_{8}$ is flux-limited and not volume-limited, but the resulting overdensity estimates have been shown to be redshiftindependent in the median (Blanton et al. 2003b). The relatively large radius of $8 h^{-1} \mathrm{Mpc}$ is chosen to provide good signal-to-noise per object, although we have shown that there is in fact more information on smaller scales (Blanton et al. 2006).

For each galaxy we estimate a second environment measure by finding the transverse distance $D_{\mathrm{cl}}$ to the nearest Virgo-like (or greater) galaxy cluster that is within $1000 \mathrm{~km} \mathrm{~s}^{-1}$ in radial distance, divided by the virial radius $R_{\text {vir }}$ of the cluster. The galaxy clusters are $\geq 10$ - 
member clusters taken from a friends-of-friends cluster catalog constructed from SDSS Main Sample galaxies with absolute magnitudes $M_{0.1_{r}}<-19.9 \mathrm{mag}$ (Berlind et al. 2006). The 10-member limit was chosen here because, after converting $M_{B}$ approximately to $M_{0.1}$, we find about 13 galaxies in Virgo at this limit (Trentham \& Hodgkin 2002). Cluster abundance as a function of multiplicity is used to convert multiplicity to mass (Berlind et al. 2006) and the mass is used to compute a virial radius $R_{\text {vir }}$ at which the cluster represents an overdensity of 200 .

On the smallest scales, we measure galaxy environment in a third way by the transverse nearest-neighbor distance $r_{\mathrm{p}, \min }$. This is defined here to be the transverse proper distance $r_{\mathrm{p}}$ to the closest neighbor galaxy in the SDSS spectroscopic sample with ${ }^{0.1} i$-band absolute magnitude $M_{0.1 i}$ brighter than $-20.0 \mathrm{mag}$ and within $200 \mathrm{~km} \mathrm{~s}^{-1}$ in line-of-sight velocity. In some cases, the "nearest" neighbor will actually be the second-nearest, because the SDSS spectrograph cannot simultaneously take two spectra closer than 55 arcsec; however, the nearest-neighbor distance $r_{\mathrm{p}, \min }$ we calculate is monotonically related to the true nearestneighbor distance statistically, at least. Besides, and as we show below, for about 45 percent of the SDSS sky region, overlapping spectroscopic pointings ameliorate or remove this constraint.

As in previous work (Quintero et al. 2004), we limit targets to redshifts $z>0.05$ to mitigate the issues of interpreting spectra taken through a small ( 3 arcsec diameter) aperture on low-redshift (and thus large in angle) galaxies. In addition, when using the clustocentric distance $D_{\mathrm{cl}}$, targets are limited to the redshifts $z<0.10$ because the cluster catalog is limited at redshift $z=0.10$. When using the nearest-neighbor distance $r_{\mathrm{p}, \min }$, targets are limited to redshifts $z<0.10$ because redshift $z=0.1$ is the redshift at which a galaxy with absolute magnitude $M_{0.1 i}=-20.0$ mag approaches the flux limit of the SDSS Main Sample.

As mentioned above, there is a technical constraint on the SDSS spectrographs such that they are unable to simultaneously take spectra of two galaxies closer than 55 arcsec, which corresponds to proper transverse distances of 38 and $71 h^{-1} \mathrm{kpc}$ at redshifts 0.05 and 0.1 in our standard $\left(\Omega_{\mathrm{M}}, \Omega_{\Lambda}\right)=(0.3,0.7)$ cosmological model. In detail, this constraint (known as "fiber collisions") affects the individual environmental measures of the galaxies in the sample. However, the effect is not expected to be large on the results for several reasons: Only a few percent of SDSS galaxy targets have been affected by the fiber collisions. In the mean, our density estimators are still monotonically related to density at least as well as other available estimators; the spectrograph constraints mainly serve to add noise and make the relationship less linear. The main results of this work depend on comparison of post-starburst and other galaxies; even if the environmental measures are affected, they are unable to produce any bias in this comparison (the resolution of collisions of galaxies in the 
SDSS Main sample is performed with no regard to luminosity or color, or any other galaxy property).

Finally, and most importantly, for the results of our environmental indicator that will be most affected by the spectrosgraph constraints - the nearest neighbor distance $r_{\mathrm{p} \text {, min }}$ - we perform the analysis with the whole sample, and then again with only the 45 percent of the sample that is not affected severely by the constraint because of multiple coverage by the spectrograph; we find no significant change.

Of course it is possible to perform environment measurements using the SDSS imaging data alone (and therefore become free of the 55 arcsec constraint entirely Eisenstein 2003; Hogg et al. 2003; Blanton et al. 2005; Blake et al. 2004; Goto 2005). However, these imagingbased environmental indicators are all either much lower in signal-to-noise (if background subtraction or deprojection is properly taken into account Eisenstein 2003) or else strongly affected by projection effects (which effectively add noise - probably biased - though it is difficult to quantify). For this reason, since we are interested in very sensitive comparisons of trace populations with dominant populations, we are much better off with environment indicators using the full three-dimensional redshift survey data despite its limitations.

\section{Results}

Figure 2 shows the conditional distribution of environmental density $\rho_{8}$ as a function of A-star excess $\log _{10}(A / K)$, in detail, at each A-star excess, it shows the 5th, 25th, 50th, 75th, and 95th percentile in $\rho_{8}$. The top panel is for all galaxies with A-star excesses, and the bottom is for those characterized as low in $\mathrm{H} \alpha$, as described above. The result is not just that the median environment is similar for $\mathrm{K}+\mathrm{A}$ and star-forming galaxies; the result is that at every value of $A / K$ for which we have reasonable signal-to-noise, the full distribution of $\rho_{8}$ is indistinguishable between the two populations. This shows that between $\mathrm{H} \alpha$ and $A / K$, it is $A / K$ that best predicts the large-scale environment in which the galaxy lies.

We have made the equivalents of Figure 2 for the other environment indicators, i.e., transverse clustocentric distance $\log _{10}\left(D_{\mathrm{cl}} / R_{\mathrm{vir}}\right)$ and transverse nearest-neighbor distance $\log _{10}\left(r_{\mathrm{p}, \mathrm{min}}\right)$. In both cases, the result is the same as in Figure 2: The environment distribution is the same for star-forming and post-starburst galaxies, to within the precision of the experiment, at each value of the A-star excess.

The top panel of Figure 3 shows the fraction of galaxies classified as star-forming by the criteria described above, in the SDSS spectroscopic sample in the redshift range $0.05<z<$ 0.20 , as a function of environmental density $\rho_{8}$. The absolute fraction is not an interesting 
number, because it depends on the luminosity and redshift ranges in the sample, and on the severity of the star-formation-rate cut (indeed, the fractions show that the A-star excess cut is more severe). The bottom panel shows the fraction of galaxies classified as $\mathrm{K}+\mathrm{A}$, with scaled versions of the curves from the top panel plotted in grey. It is remarkable how well the three curves match one another. The only exception is at very low densities (recall that the mean density - mean taken over galaxies - is well above unity because galaxies are clustered), where there is a slight underdensity of $\mathrm{K}+\mathrm{A}$ galaxies relative to star-forming galaxies.

Interestingly, and somewhat beside the point of this work, there is also a tiny but significant difference visible in Figure 3 in the relative abundances of the two different kinds of star-forming galaxies ( $\mathrm{H} \alpha$ excess and A-star excess) at low environmental density $\rho_{8}$. This may have something to do with the geometry or density or abundance of gas in the star-forming galaxies in the lowest density environments.

Figure 4 is similar to Figure 3 but with transverse clustocentric distance $\log _{10}\left(D_{\mathrm{cl}} / R_{\mathrm{vir}}\right)$ acting as the environment indicator. Again it is remarkable how similar are the three curves outside of the virial radius. Inside the virial radius, the abundance of post-starburst galaxies relative to star-forming galaxies is higher. This deviation indicates that a small number of the post-starburst galaxies may be triggered by interactions with the intracluster medium. Unfortunately, the change in relative abundance is not large, and another equally valid way to express the discrepancy is that the $\mathrm{K}+\mathrm{A}$ fraction is a weaker function of environment than the star-forming fraction.

Figure 5 is similar to Figure 3 but with transverse nearest-neighbor distance $r_{\mathrm{p} \text {,min }}$ acting as the environment indicator. Even on these very small scales, the three curves are very similar. Again the exception is that there is a very slight excess of $\mathrm{K}+$ As relative to star-formers with nearby neighbors, or, the $\mathrm{K}+\mathrm{A}$ fraction is a weaker function of small-scale environment than the star-forming fraction. This is consistent with previous results on very small-scale environments of post-starburst galaxies Goto (2005).

As mentioned above, the nearest-neighbor distance $r_{\mathrm{p}, \mathrm{min}}$ can in principle be affected by the SDSS spectrograph 55 arcsec constraint. For this reason, we have re-made Figure 3 using only galaxies inside SDSS "overlap regions" where the spectrograph had multiple opportunities to obtain spectra in the same sky location, and therefore "picked up" most of the galaxies excluded by the 55 arcsec constraint. Roughly 45 percent of the sky area used in this work is inside overlap regions, so the overlap subsample is not small. Figure 6 is the result; it is the same as Figure 5 except that it only makes use of galaxies for which the 55 arcsec constraint is not significant. The great similarity of the two Figures demonstrates that our results are not being strongly affected by this constraint, as expected. 


\section{Discussion}

As we have discussed elsewhere (Quintero et al. 2004), post-starburst galaxies plausibly lie on an evolutionary sequence between disk-dominated galaxies, which are forming stars and contain the neutral gas fuel for further star formation, and bulge-dominated galaxies, which have no star-formation fuel and show chemical signatures of past star-formation bursts. Poststarburst galaxies might even be the remnants of major mergers. A priori, the environments of these galaxies could be either like those of disk-dominated galaxies or those of bulgedominated galaxies, or somewhere in-between. Of course, prior to this study, it was already known that the mean environments of post-starburst galaxies are more similar to those of disk-dominated galaxies than those of bulge-dominated galaxies (Zabludoff et al. 1996; Quintero et al. 2004; Blake et al. 2004, Helmboldt et al., in preparation). Here we have not only confirmed this result, we have shown that for each value of the A-star excess, the post-starburst galaxies with that A-star excess find themselves in similar environments to star-forming galaxies with that same A-star excess. In other words, A-star excess is more closely related to environment than $\mathrm{H} \alpha \mathrm{EW}$, since the post-starburst galaxies have $\mathrm{H} \alpha \mathrm{EWs}$ like bulge-dominated galaxies.

This result confirms our "null hypothesis" that the processes that connect a galaxy's star-formation history to its environment act on long timescales, longer than A-star lifetimes $(\sim 1 \mathrm{Gyr})$. This is not surprising, since at typical cosmological velocities $\left(\sim 100 \mathrm{~km} \mathrm{~s}^{-1}\right)$, a galaxy can only travel $\sim 1 \mathrm{Mpc}$ in a Gyr, and there is now pretty good empirical evidence that everything important about galaxy environments happens on scales $<1 \mathrm{Mpc}$ (Blanton et al. 2006).

On the other hand, this result is somewhat difficult to reconcile with the observation from chemical abundances (e.g., Worthey 1998; Eisenstein et al. 2003) and central stellar densities (Quintero et al. 2004) that a typical red (early-type, or bulge-dominated) galaxy has undergone a brief but strong starburst at some point in its past. Perhaps the starbursts are triggered very randomly, with just a slight change in starburst trigger probability with environment, so we don't see the relation clearly. Perhaps the observed relationship between star-formation rate and environment was set down at very high redshift and is in fact diluting at the present epoch. Perhaps the starbursts are primarily triggered prior to infall into dense

environments, in which case the galaxies somehow "know" the environment in which they will end up!

We have shown that the fraction of the whole SDSS galaxy sample classified as " $\mathrm{K}+\mathrm{A}$ " (post-starburst) is a function of environment, and that its dependence on environment is very similar to that of the fraction of the sample classified as star-forming. This is also consistent with our null hypothesis. The only deviations all have the sense that the $\mathrm{K}+\mathrm{A}$ fraction is 
a slightly weaker function of environment than the star-forming fraction: We find slightly fewer $\mathrm{K}+\mathrm{As}$ in extremely low density environments, and slightly more inside the virial radii of massive clusters and close to luminous neighbors than we would expect by naive scaling of the star-forming fraction.

None of these deviations from the predictions of the null hypothesis are large, but they may point to triggering mechanisms for the starburst and post-starburst phases: It is possible that a small fraction of post-starburst galaxies are triggered by close passages of luminous galaxies. It seems likely, from Figure 4, that a small fraction of post-starbursts are triggered by interactions with intracluster medium, because the deviation of the post-starburst fraction relative to the star-forming fraction does occur at the virial radius.

We can therefore make two negative statements about the triggering of the majority of starbursts, both plausible at the outset: The first is that only a small fraction of poststarburst galaxies are triggered by external tidal impulses from close passages of massive galaxies. This does not rule out the possibility that they are created by mergers or accretion events, but if they are, the truncation of star-formation must occur after the two merging galaxies are no longer identifiable as separate galaxies. Our punchline may seem to be at odds with the punchline of some previous work (Goto 2005). Quantitatively, however, there is no disagreement, given the differences in methodology, and in fact both studies do find small excesses of neighbors at small scales. This suggests that although tidal-impulse triggering (i.e., triggering by close passages of massive neighbors) no-doubt occurs, it is not the dominant mechanism.

The second negative statement we can make is that only a small fraction of poststarburst galaxies are created by IGM interactions on infall into clusters. Certainly some are; the identification of the virial radius in the abundance of post-starburst galaxies relative to star-forming galaxies is intriguing. The galaxy populations inside clusters are very different in morphological mix and star-formation-history mix than galaxy populations elsewhere. What physical process are involved in enforcing these differences? Many have hypothesized - quite naturally - that radical transformations must happen on infall (Poggianti et al. 1999; Balogh et al. 2000; Kodama \& Bower 2001); indeed some galaxies have been "caught in the act" of a radical transformation (e.g., Vogt et al. 2004). Quantitatively, however, the fraction of galaxies showing clear evidence for intracluster medium interactions is very small. This is consistent with prior work in this area (Poggianti et al. 1999; Balogh et al. 2000; Kodama \& Bower 2001; Vogt et al. 2004).

The orbital time for a galaxy falling into a cluster is only a few times longer than the lifetime of A stars, so the excess of post-starburst galaxies in the infall regions is expected to "smear" into the cluster center; i.e., the morphology of Figure 4 is about right (given 
the small numbers) for the intra-cluster medium hypothesis. What remains to be seen is whether, quantitatively, the tiny number of post-starburst galaxies observed within clusters is consistent with the competing facts that clusters are continuously growing by accretion of field galaxies and groups and that the morphological and star-formation mix in clusters is different inside and outside of clusters; the prodigious expected infall means that a lot of galaxies ought to be changing their morphologies and star-formation rates on infall. We expect such an analysis - beyond the scope of this paper - to conclude that either the transformation process is slow ( $>1 \mathrm{Gyr}$, which is not necessarily consistent with observations of abundances in bulge-dominated galaxies), or else that the galaxies in clusters somehow "knew in advance" (from their pre-infall group or filament environment) that they were destined to end up in a cluster. A philosophical point arises here: The fact that galaxy populations inside clusters are different from those outside does not necessarily mean that transformations take place as galaxies move from one environment to the other.

The remaining hypotheses for the triggering of the starburst (or, more properly, starformation truncation) events that precedes the post-starburst phases of these galaxies are: some kinds of random internal catastrophes or some kinds of galaxy-galaxy mergers. This latter possibility, which is consistent with all of the results here, is directly supported by the discovery of post-merger morphological signatures (e.g., tidal arms) in many post-starburst galaxies (Yang et al. 2004; Goto 2005). It is also exciting, because merging is one of the fundamental processes of cosmogony, and holds great promise for providing precise connections between cosmological observations and theory at small scales.

It is a pleasure to thank Tomo Goto, Joe Helmboldt, Don Schneider, Michael Strauss, Douglas Tucker, and an anonymous referee for useful comments on the manuscript. DWH and MM were generously hosted by the Massachusetts Institute of Technology Kavli Institute for Astrophysics and Space Research during the period of this research. DWH, MM, MRB, and ADQ are partially supported by the National Aeronautics and Space Administration (NASA; grant NAG5-11669); DWH, MM, and MRB are partially supported by the National Science Foundation (NSF; grant AST-0428465). This research made use of the NASA Astrophysics Data System. It also made use of the "idlutils" codebase maintained by David Schlegel, Doug Finkbeiner, and others.

Funding for the creation and distribution of the SDSS Archive has been provided by the Alfred P. Sloan Foundation, the Participating Institutions, NASA, the NSF, the U.S. Department of Energy, the Japanese Monbukagakusho, and the Max Planck Society. The SDSS Web site is "http://www.sdss.org/".

The SDSS is managed by the Astrophysical Research Consortium for the Participating 
Institutions. The Participating Institutions are The University of Chicago, Fermilab, the Institute for Advanced Study, the Japan Participation Group, The Johns Hopkins University, the Korean Scientist Group, Los Alamos National Laboratory, the Max-Planck-Institute for Astronomy, the Max-Planck-Institute for Astrophysics, New Mexico State University, University of Pittsburgh, University of Portsmouth, Princeton University, the United States Naval Observatory, and the University of Washington.

\section{REFERENCES}

Abazajian, K. et al. 2003, AJ, 126, 2081

Abazajian, K. et al. 2004, AJ, 128, 502

Balogh, M. L., Christlein, D., Zabludoff, A. I., \& Zaritsky, D. 2001, ApJ, 557, 117

Balogh, M. L., Navarro, J. F., \& Morris, S. L. 2000, ApJ, 540, 113

Bell, E. F., Wolf, C., Meisenheimer, K., Rix, H.-W., Borch, A., Dye, S., Kleinheinrich, M., Wisotzki, L., \& McIntosh, D. H. 2004, ApJ, 608, 752

Berlind, A. A., Frieman, J. A., Weinberg, D. H., Blanton, M. R., Warren, M., Abazajian, K., Scranton, R., Hogg, D. W., \& Scoccimarro, R. 2006, ApJ, submitted (astro$\mathrm{ph} / 0601346)$

Blake, C. et al. 2004, MNRAS, 355, 713

Blanton, M. R. 2006, ApJ, in press (astro-ph/0512127)

Blanton, M. R., Brinkmann, J., Csabai, I., Doi, M., Eisenstein, D. J., Fukugita, M., Gunn, J. E., Hogg, D. W., \& Schlegel, D. J. 2003, AJ, 125, 2348

Blanton, M. R., Eisenstein, D., Hogg, D. W., Schlegel, D. J., \& Brinkmann, J. 2005, ApJ, 629,143

Blanton, M. R., Lin, H., Lupton, R. H., Maley, F. M., Young, N., Zehavi, I., \& Loveday, J. 2003a, AJ, 125, 2276

Blanton, M. R. et al. 2003b, ApJ, 594, 186

Blanton, M. R. et al. 2003c, ApJ, 592, 819

Blanton, M. R. et al. 2006, ApJ, in press (astro-ph/0411037) 
Brown, M. J. I. et al. 2006, ApJ, submitted

Butcher, H. R. \& Oemler, A. 1978, ApJ, 219, 18

Couch, W. J. \& Sharples, R. M. 1987, MNRAS, 229, 423

Cowie, L. L., Songaila, A., \& Barger, A. J. 1999, ApJ, 118, 603

Dressler, A. 1980, ApJ, 236, 351

Dressler, A. \& Gunn, J. E. 1983, ApJ, 270, 7

Dressler, A., Smail, I., Poggianti, B. M., Butcher, H., \& Oemler, A. J. 1999, ApJS, 122, 51

Eisenstein, D. J. 2003, ApJ, 586, 718

Eisenstein, D. J. et al. 2001, AJ, 122, 2267

Eisenstein, D. J. et al. 2003, ApJ, 585, 694

Faber, S. M. et al. 2006, ApJ, submitted, (astro-ph/0506044)

Flores, H., Hammer, F., Thuan, T. X., Césarsky, C., Desert, F. X., Omont, A., Lilly, S. J., Eales, S., Crampton, D., \& Le Fèvre, O. 1999, ApJ, 517, 148

Franx, M. 1993, ApJ, 407, L5

Fukugita, M., Ichikawa, T., Gunn, J. E., Doi, M., Shimasaku, K., \& Schneider, D. P. 1996, AJ, 111, 1748

Gomez, P. et al. 2003, ApJ, 584, 210

Goto, T. 2004, A\&A, 427, 125

Goto, T. 2005, MNRAS, 357, 937

Gunn, J. E., Carr, M. A., Rockosi, C. M., Sekiguchi, M., et al. 1998, AJ, 116, 3040

Haarsma, D. B., Partridge, R. B., Windhorst, R. A., \& Richards, E. A. 2000, ApJ, 544, 641

Hammer, F., Flores, H., Lilly, S. J., Crampton, D., Le Fevre, O., Rola, C., Mallen-Ornelas, G., Schade, D., \& Tresse, L. 1997, ApJ, 481, 49

Hashimoto, Y., Oemler, A., Lin, H., \& Tucker, D. L. 1998, ApJ, 499, 589

Hashimoto, Y. \& Oemler, A. J. 1999, ApJ, 510, 609 
Hogg, D. W. 1999, astro-ph/9905116

Hogg, D. W., Cohen, J. G., Blandford, R., \& Pahre, M. A. 1998, ApJ, 504, 622

Hogg, D. W. et al. 2003, ApJ, 585, L5

Hogg, D. W. et al. 2004, ApJ, 601, L29

Ivezić, Ž. et al. 2004, Astronomische Nachrichten, 325, 583

Juneau, S. et al. 2005, ApJ, 619, L135

Kauffmann, G., White, S. D. M., Heckman, T. M., Ménard, B., Brinchmann, J., Charlot, S., Tremonti, C., \& Brinkmann, J. 2004, MNRAS, 314

Kennicutt, R. C. 1983, AJ, 88, 483

Kodama, T. \& Bower, R. G. 2001, MNRAS, 321, 18

Lewis, I. et al. 2002, MNRAS, 334, 673

Lilly, S. J., Le Fevre, O., Hammer, F., \& Crampton, D. 1996, ApJ, 460, L1

Lupton, R. H., Gunn, J. E., Ivezić, Z., Knapp, G. R., Kent, S., \& Yasuda, N. 2001, in ASP Conf. Ser. 238: Astronomical Data Analysis Software and Systems X, Vol. 10, 269

Martínez, H. J., Zandivarez, A., Domínguez, M., Merchán, M. E., \& Lambas, D. G. 2002, MNRAS, 333, L31

Mobasher, B., Cram, L., Georgakakis, A., \& Hopkins, A. 1999, MNRAS, 308, 45

Pier, J. R., Munn, J. A., Hindsley, R. B., Hennessy, G. S., Kent, S. M., Lupton, R. H., \& Ivezić, Ž. 2003, AJ, 125, 1559

Poggianti, B. M., Smail, I., Dressler, A., Couch, W. J., Barger, A. J., Butcher, H., Ellis, R. S., \& Oemler, A. 1999, ApJ, 518, 576

Poggianti, B. M. \& Wu, H. 2000, ApJ, 529, 157

Postman, M. \& Geller, M. 1984, ApJ, 281, 95

Quintero, A. D., Berlind, A. A., Blanton, M. R., \& Hogg, D. W. 2006, ApJ, submitted (astro-ph/0512004)

Quintero, A. D. et al. 2004, ApJ, 602, 190 
Richards, G. et al. 2002, AJ, 123, 2945

Rowan-Robinson, M. et al. 1997, MNRAS, 289, 490

Schiminovich, D. et al. 2005, ApJ, 619, L47

Schlegel, D. J., Finkbeiner, D. P., \& Davis, M. 1998, ApJ, 500, 525

Smith, J. A., Tucker, D. L., et al. 2002, AJ, 123, 2121

Stoughton, C. et al. 2002, AJ, 123, 485

Strauss, M. A. et al. 2002, AJ, 124, 1810

Trentham, N. \& Hodgkin, S. 2002, MNRAS, 333, 423

Tresse, L. \& Maddox, S. J. 1998, ApJ, 495, 691

Vogt, N. P., Haynes, M. P., Giovanelli, R., \& Herter, T. 2004, AJ, 127, 3300

Worthey, G. 1998, PASP, 110, 888

Yang, Y., Zabludoff, A. I., Zaritsky, D., Lauer, T. R., \& Mihos, J. C. 2004, ApJ, 607, 258

York, D. et al. 2000, AJ, 120, 1579

Zabludoff, A. I., Zaritsky, D., Lin, H., Tucker, D., Hashimoto, Y., Shectman, S. A., Oemler, A., \& Kirshner, R. P. 1996, ApJ, 466, 104 


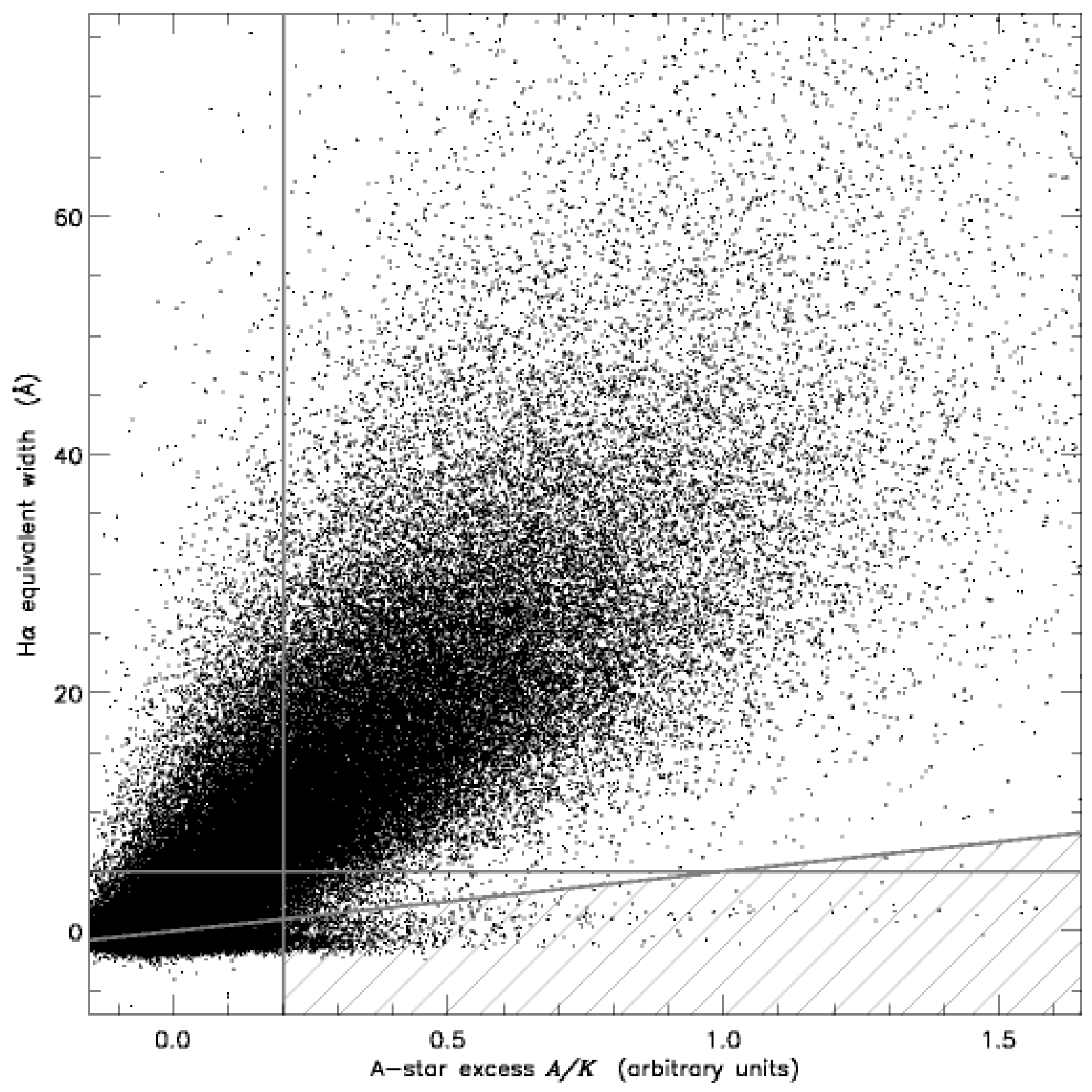

Fig. 1. - The $\mathrm{H} \alpha \mathrm{EW}$ and A-star excess $[A / K]$ measurements for the sample galaxies. Galaxies above the horizontal line are deemed "star-forming" by $\mathrm{H} \alpha \mathrm{EW}$, galaxies right of the vertical line are deemed "star-forming" by A-star excess, and galaxies in the hatched region are deemed "K+A". Galaxies below the sloped line are deemed "low $\mathrm{H} \alpha$ ". 


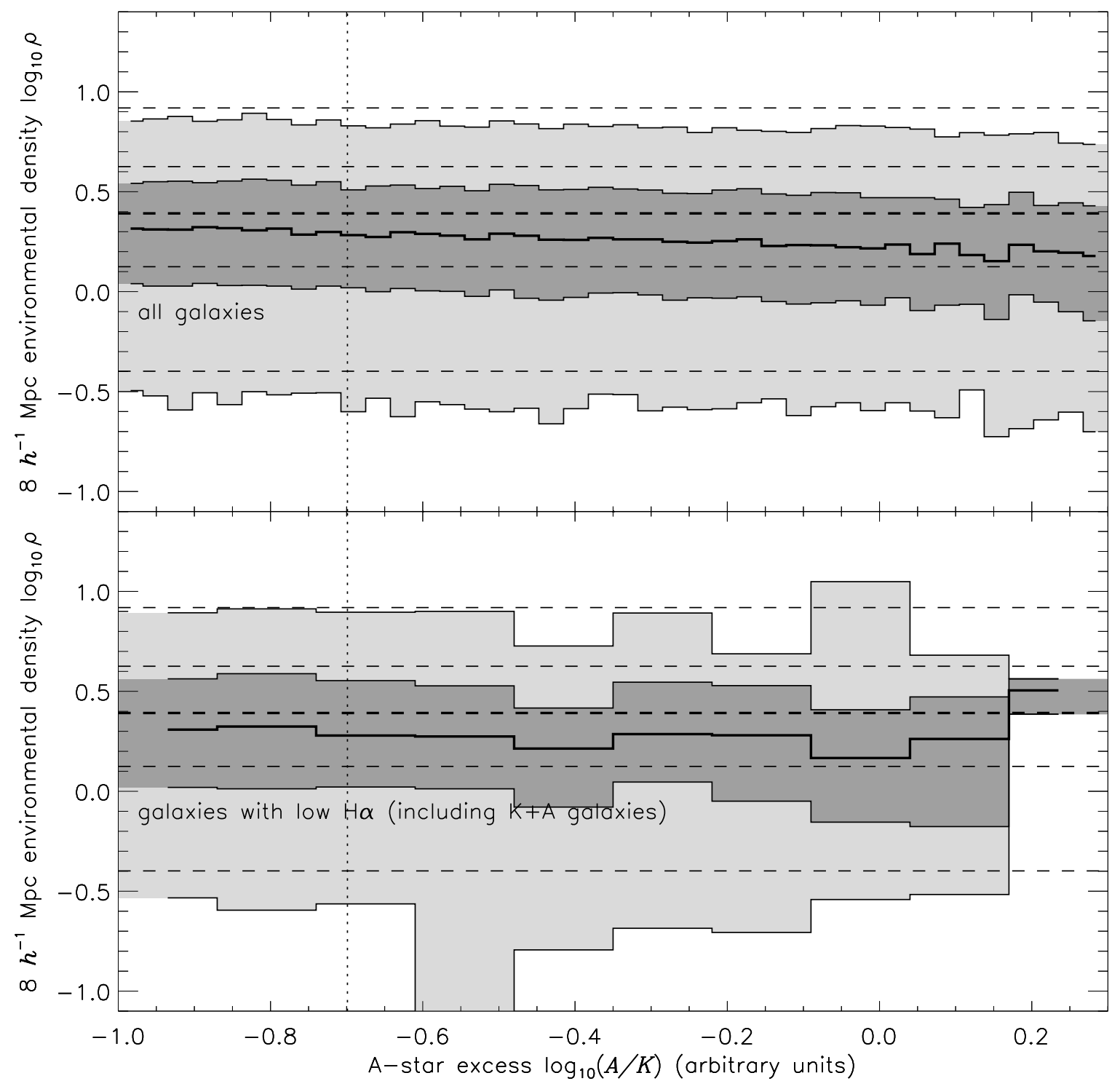

Fig. 2.- The density distribution conditioned on A-star excess. The five lines show the 5, $25,50,75$, and 95 percentiles in density $\rho_{8}$ (normalized to cosmic mean density) of galaxies in $8 h^{-1}$ Mpc radius spheres in comoving redshift space around target galaxies as a function of the A-star excess $[A / K]$ in the target galaxies. The top panel is for all galaxies in the sample, and the bottom is for those deficient in $\mathrm{H} \alpha$ relative to the A-star excess (see text for details); the post-starburst or $\mathrm{K}+\mathrm{A}$ galaxies are in the right side of the lower panel. See text for details of the density and A-star excess measurements. The horizontal dashed lines show the same percentiles but for galaxies with no A-star excess, and the vertical dotted line is the minimum A-star excess required for a galaxy to be classified as star-forming (top panel) or $\mathrm{K}+\mathrm{A}$ (bottom panel) in this work. Note that both populations have very similar dependences of environmental quantiles on A-star excess. 


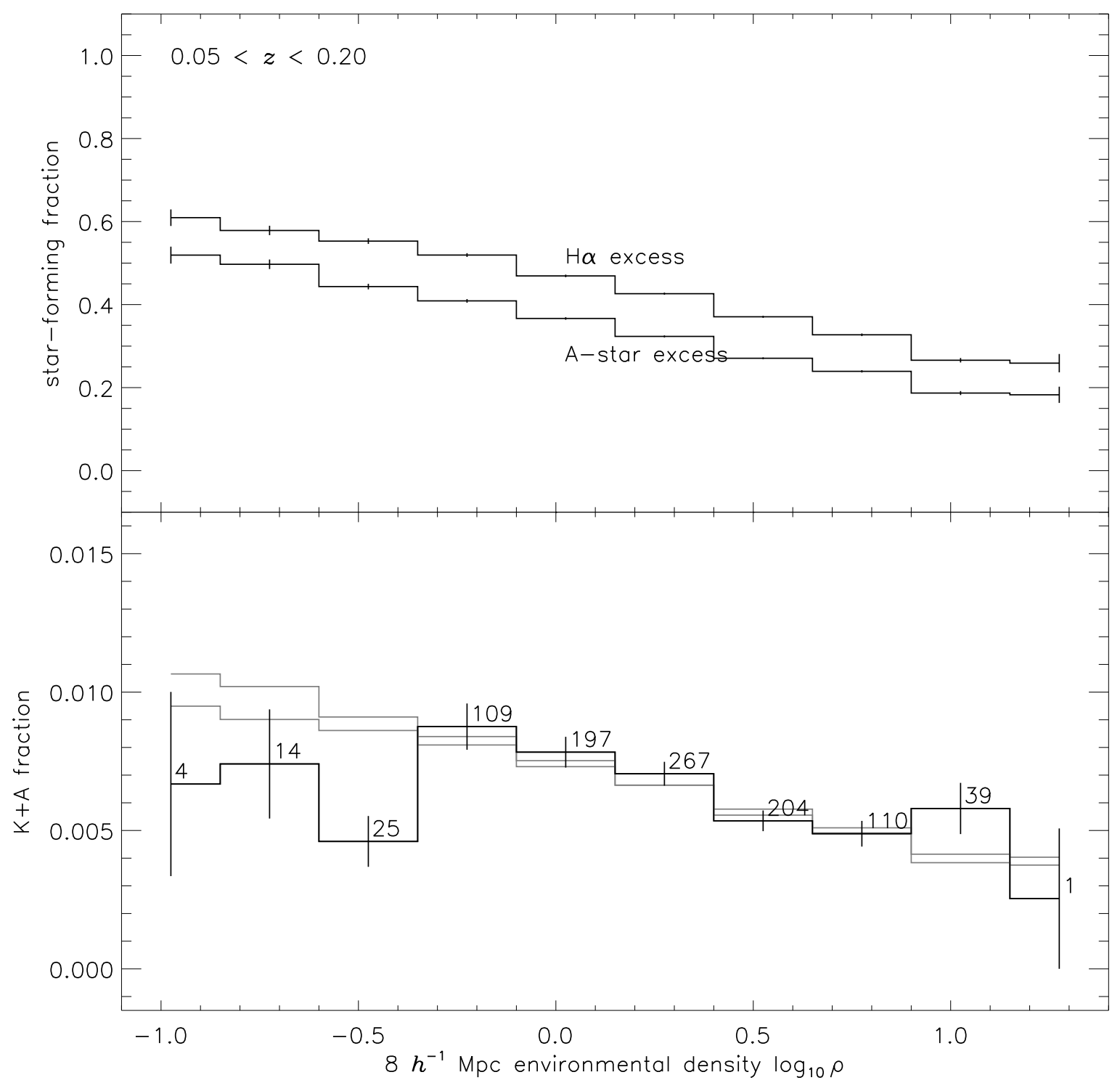

Fig. 3.- The fractional abundance of galaxy populations as a function of environmental density. The top panel shows the fraction of galaxies deemed "star forming" by $\mathrm{H} \alpha \mathrm{EW}$ (top curve) and by A-star excess $[A / K]$ (bottom curve). The vertical offset of the curves comes from the fact that the A-star excess cut is more severe than the $\mathrm{H} \alpha \mathrm{EW}$ cut (see text for details). The bottom panel shows the same but for the galaxies deemed post-starburst ( $\mathrm{K}+\mathrm{A}$; see text for definition). Each histogram bin is labeled by the number of $\mathrm{K}+\mathrm{A}$ galaxies in that bin. Also shown in the bottom panel are scaled versions of the curves from the top panel, scaled by the mean abundance ratio. 


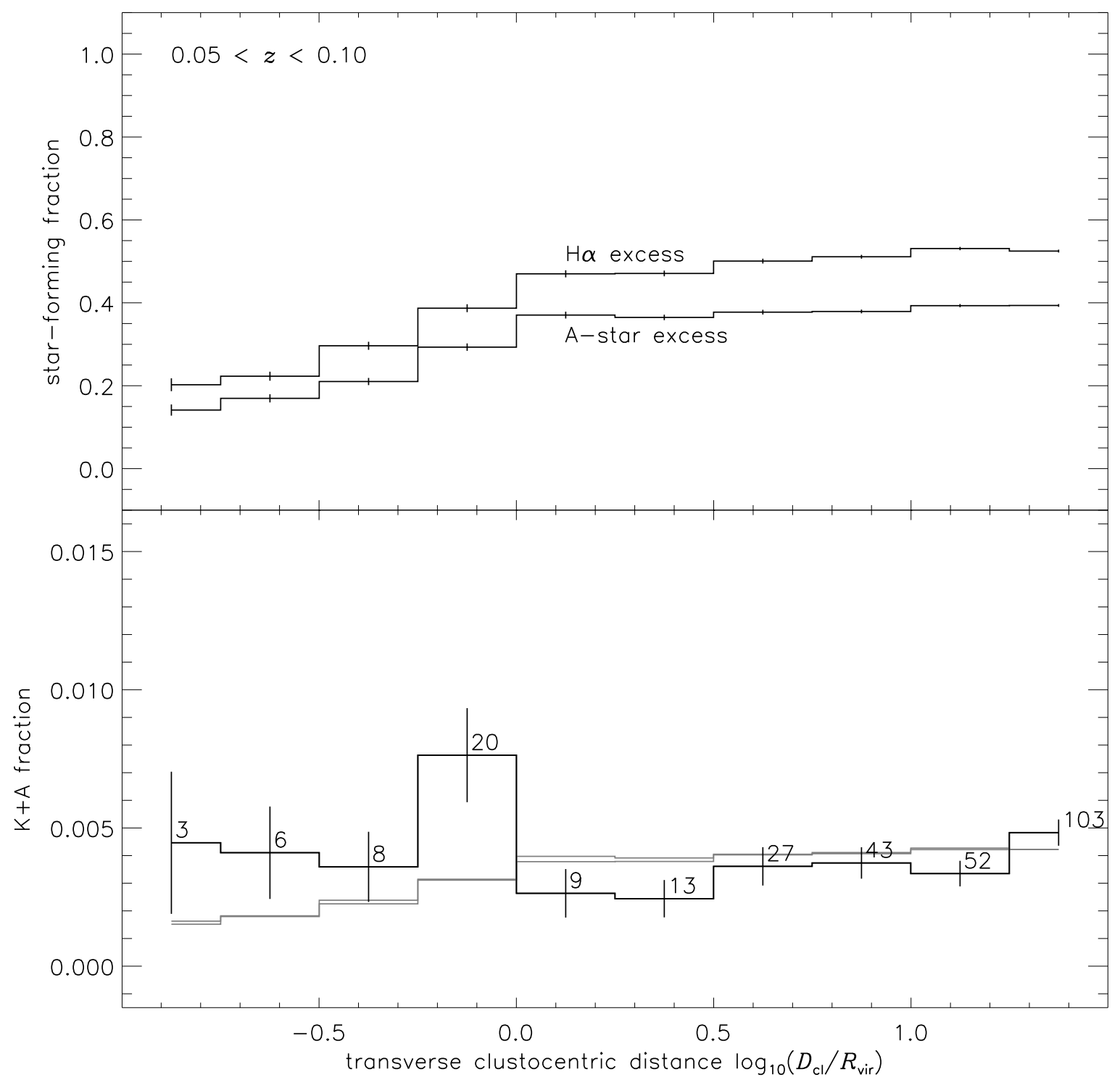

Fig. 4. - Same as Figure 3, except using the transverse, virial-scaled clustocentric distance $D_{\mathrm{cl}} / R_{\text {vir }}$ (see text) as the environment indicator. 


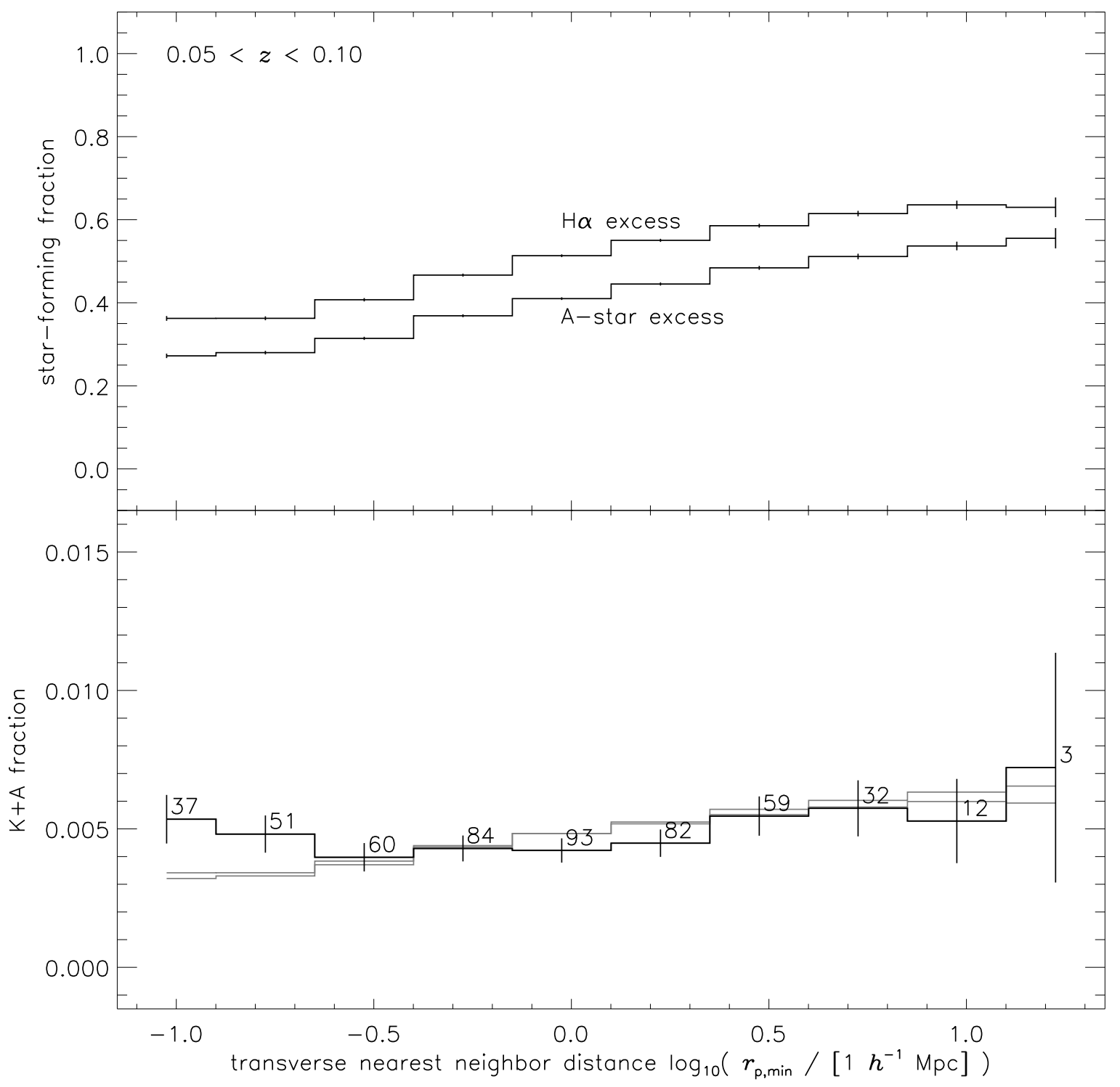

Fig. 5.- Same as Figure 3, except using the transverse, proper, nearest-neighbor distance $r_{\mathrm{p}, \min }$ (see text) as the environment indicator. 


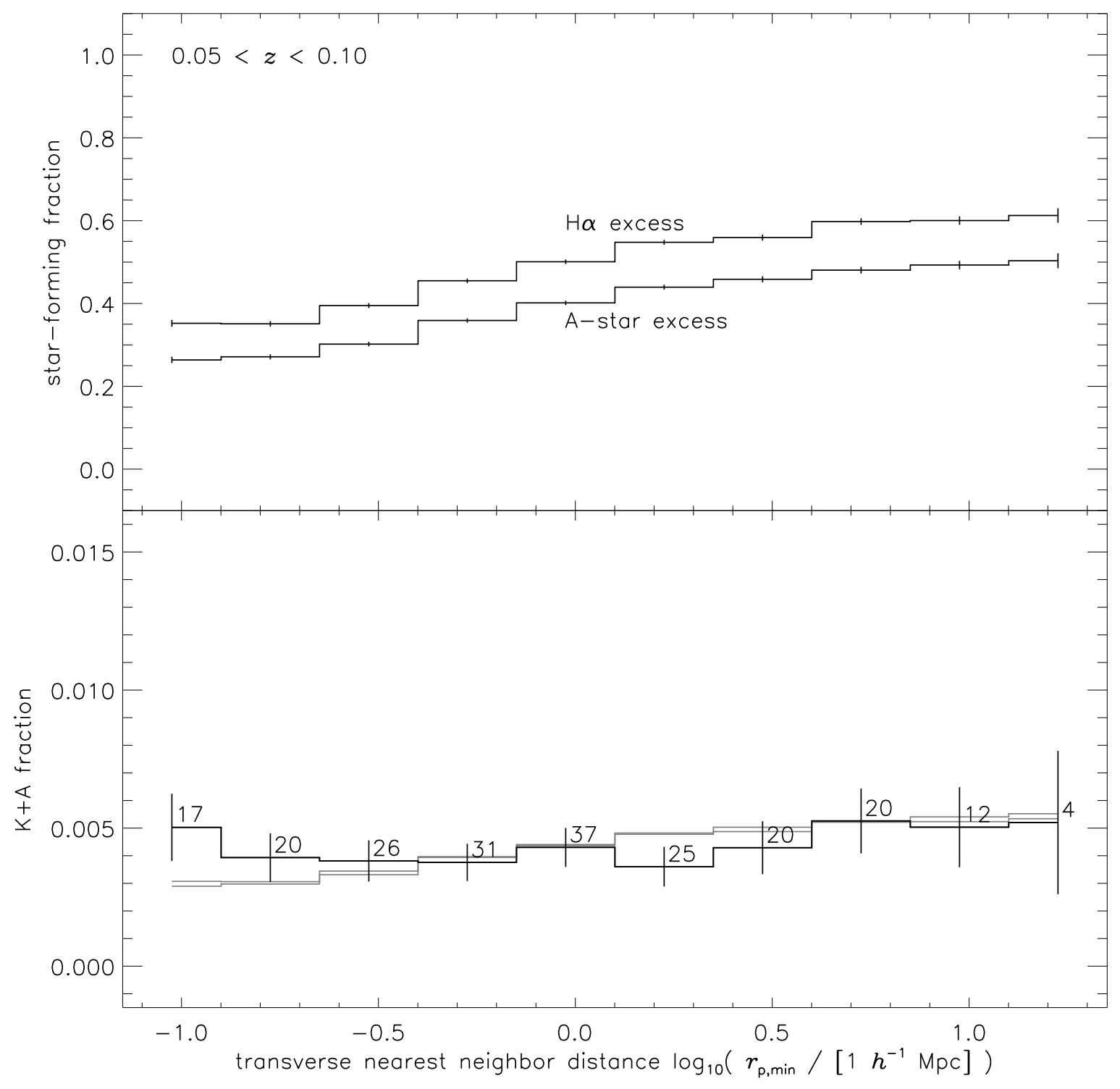

Fig. 6.- Same as Figure 5, but using only galaxies within the SDSS "overlap" regions, where the 55 arcsec spectrograph constraint does not apply. The similarity to Figure 5 demonstrates that spectrograph constraints are not significantly affecting the figure. 